\title{
Percutaneous tricuspid valve implantation in failing bioprosthesis
}

\author{
Andreas Eicken, Peter Ewert \\ Department of Pediatric Cardiology and Congenital Heart Disease, German Heart Centre Munich, Technische Universität Munich, Munich, Germany \\ Contributions: (I) Conception and design: All authors; (II) Administrative support: All authors; (III) Provision of study materials or patients: All \\ authors; (IV) Collection and assembly of data: All authors; (V) Data analysis and interpretation: All authors; (VI) Manuscript writing: All authors; (VII) \\ Final approval of manuscript: All authors. \\ Correspondence to: Andreas Eicken, MD, FESC. German Heart Centre Munich, Lazarettstr 36, Munich 80636, Germany. Email: eicken@dhm.mhn.de.
}

\begin{abstract}
Severe tricuspid valve (TV) dysfunction in patients with congenital heart disease (CHD) is usually treated by open heart surgery in relatively young patients. If a valve plasty is not possible, a biological valve is implanted with a limited durability. Due to valve degeneration repeated valve exchanges are necessary in these patients. To expand the lifetime of a bioprosthesis in tricuspid position percutaneous TV implantation (PTVI) was introduced recently. PTVI is a promising new catheter interventional technology. The current review summarizes the indication for PTVI, describes the procedure itself and gives an outlook on medium to long-term results of this catheter intervention. PTVI in patients with severe TV dysfunction is less invasive, safe and effective, if performed by an experienced operator, and may help to reduce the total number of open-heart surgeries during a patient's life time. However, further studies with larger patient numbers and longer follow-up are needed.
\end{abstract}

Keywords: Tricuspid valve (TV); transcatheter valve replacement; valve-in-valve replacement

Submitted Jun 26, 2018. Accepted for publication Aug 14, 2018.

doi: $10.21037 / \mathrm{cdt} .2018 .09 .08$

View this article at: http://dx.doi.org/10.21037/cdt.2018.09.08

\section{Introduction}

Severe tricuspid valve (TV) dysfunction (TV regurgitation, TR; TV stenosis, TS, a combination of both) may have different etiologies. Primary cause can be congenital, as in Ebstein's anomaly of the TV, where tethering of the septal TV leaflet leads to downward orientation of the TV annulus (1). This results in various degrees of TR, an atrialized segment of the right ventricle (RV) and consecutive left ventricular dysfunction. If a communication at atrial level is present, right to left shunt may induce severe cyanosis. Secondly, severe TR may be a symptom of various left heart pathologies, finally leading to chronic pressure overload of the RV, causing RV, and concomitant TV-ring-dilatation resulting in functional TR with poor prognosis (2-4). In these patients, TR is one component in a complex cardiac disease and a marker of an advanced disease stage. Finally, TV dysfunction may be present after previous surgery or catheter intervention for treatment of various congenital cardiac anomalies.

Patients with severe TV dysfunction may develop right ventricular failure and, hence, require treatment (5). The initial treatment option is TV surgery. For Ebstein's anomaly, excellent results have been published with conservative operative techniques and more recently by the Cone-operation, pioneered by da Silva et al. $(6,7)$. In patients with functional TR a thoughtful risk benefit analysis must be done, since abolishment of TR is not curative if left heart failure is the leading cause. If surgical tricuspid valvuloplasty is not feasible or the result of this operation is not satisfactory, TV replacement has to be performed $(8,9)$. In most of these patients a bioprosthesis is implanted in TV position. With elapsing time, biological valves undergo degeneration leading to TV regurgitation and/or stenosis, right atrial (RA) enlargement, onset of clinical deterioration, atrial arrhythmias and right heart failure (10). In these patients the TVs need to be replaced again (11). 


\section{Percutaneous valve therapy}

Patients with a failing bioprosthesis in tricuspid position are potentially excellent candidates for percutaneous TV implantation (PTVI). Percutaneous valve therapy was pioneered by Bonhoeffer et al. nearly 20 years ago (12), when a bovine jugular vein valve, sewn into a Cheatham platinum stent, was implanted from the groin into a stenotic bioprosthesis (Carpentier-Edwards bioprosthesis) in the right ventricular outflow tract of a child. Percutaneous pulmonary valve implantation (PPVI) preceded transcatheter aortic valve replacement (TAVR) by several years and the excellent PPVI results paved the way for TAVR, which is now performed in large numbers worldwide. The initial intention of PPVI was to prolong the life-span of a surgically implanted bioprosthesis by at least 2 years with the final goal to reduce the total number of open-heart surgeries in patients with CHD over their lifetime. The Melody valve (Medtronic, Minneapolis, MN, USA) received the CE mark for PPVI in Canada and Europe in 2006 and FDA approval occurred in 2010. The Sapien XT valve (Edwards, Irvine, CA, USA) was approved by the FDA for percutaneous implantation in pulmonic position in the year 2016.

However, both valves are not approved for implantation in tricuspid position, hence, PTVI currently is an offlabel catheter intervention. This needs to be discussed with patients-and at present with health care insurances. The failing bioprosthesis in TV position usually is an ideal landing zone for a percutaneously implanted valve, if the dimensions are well defined and the valve size is adequately selected. So far, an increasing number of patients have been treated by PTVI and longer follow-up is gathered in PTVIregistries (13-20).

To avoid registries open heart-surgery with its known risks and co-morbidities and to reduce the total number of surgical procedures during a patient's life-span, PTVI is, in our view, a promising alternative treatment option.

\section{PTVI}

The intervention is carried out in general anesthesia, or in deep conscious sedation. It has been described previously $(15,21)$. After vascular access is achieved for both the femoral vein and artery a complete diagnostic catheterization is performed. If femoral veins are occluded, a jugular venous access may be used. Because accurate valve delivery requires coaxial positioning within the bioprosthesis, the $\mathrm{X}$-ray system is rotated so that the imaging plane is aligned parallel to the radiopaque valve ring (if present) in the antero-posterior camera. The diastolic inflow gradient between the right atrium (RA) and the end-diastolic right ventricular pressure (RVEDP) is determined. The degree of tricuspid regurgitation is assessed by a right ventricular angiogram. A weight adjusted dose of heparin (100 units $/ \mathrm{kg}$, max. 5,000 units) is given. Periprocedural treatment includes antibiotic prophylaxis with a second generation cephalosporine (cefuroxime $100 \mathrm{mg} / \mathrm{kg} / \mathrm{d}$, divided in 3 doses or $3 \times 1.5 \mathrm{~g}$ in adults).

A superstiff 0.035 -inch guide wire (Meier Wire, Boston Scientific Natick MA, USA; Lunderquist Wire, Cook Medical Bloomington IN, USA; or Amplatz Ultrastiff, Cook Medical) is positioned distally into one pulmonary artery. Although PTVI may be performed directly into a known TV bioprosthesis $(22,23)$ we prefer to inflate a noncompliant balloon $(30 \mathrm{~mm} \times 60 \mathrm{~mm})$ in the TV, if necessary with concomitant depiction of the coronary artery anatomy. For PTVI pre-stenting is only necessary in heavily calcified homografts. Depending on the assessed valve diameter a percutaneous valve (Medtronic Melody valve, usually on a $22-\mathrm{mm}$ balloon, or Edwards Sapien valve $26 \mathrm{~mm}$, or $29 \mathrm{~mm}$ ) is prepared and delivered over the super-stiff guide wire, which should stay in the pulmonary artery. In our practice, a Melody ${ }^{\circledR}$ valve (Medtronic Inc. Minneapolis $M N$, USA), was chosen if the internal valve diameter was $<24 \mathrm{~mm}$. For larger internal valve diameters, the Sapien 26 or Sapien XT 29 (Edwards Lifesciences Corp., Irvine CA, USA) valves were used. Recently, only the Sapien S3 valve is available at our centre. Strict perpendicular positioning of the $\mathrm{X}$-ray system enables alignment of the transcatheter valve so that the proximal struts just protrude out of the bioprosthesis into the RA clearly across the rigid valve ring. Positioning too far into the bioprosthesis, distal to the rigid valve ring, may increase the risk of valve embolization into the RV. If a Sapien valve is used, the valve landing zone has to be circular and the diameter needs to be close to the nominal outer diameter of the used Sapien valve. If the valve is not completely dilated close to the nominal outer valve diameter, valve dysfunction may occur (stenosis or regurgitation). It may be necessary to re-dilate the valve with ultra-high-pressure balloons (Atlas gold Bard, Tempe, AZ, USA). If this is necessary, rapid pacing may be helpful to stabilize the balloon in the valve. Finally, the diastolic inflow gradient (RA-RV) is reassessed and a $\mathrm{RV}$ angiogram documents the result. On the following day the result is controlled by echocardiography. Aspirin 

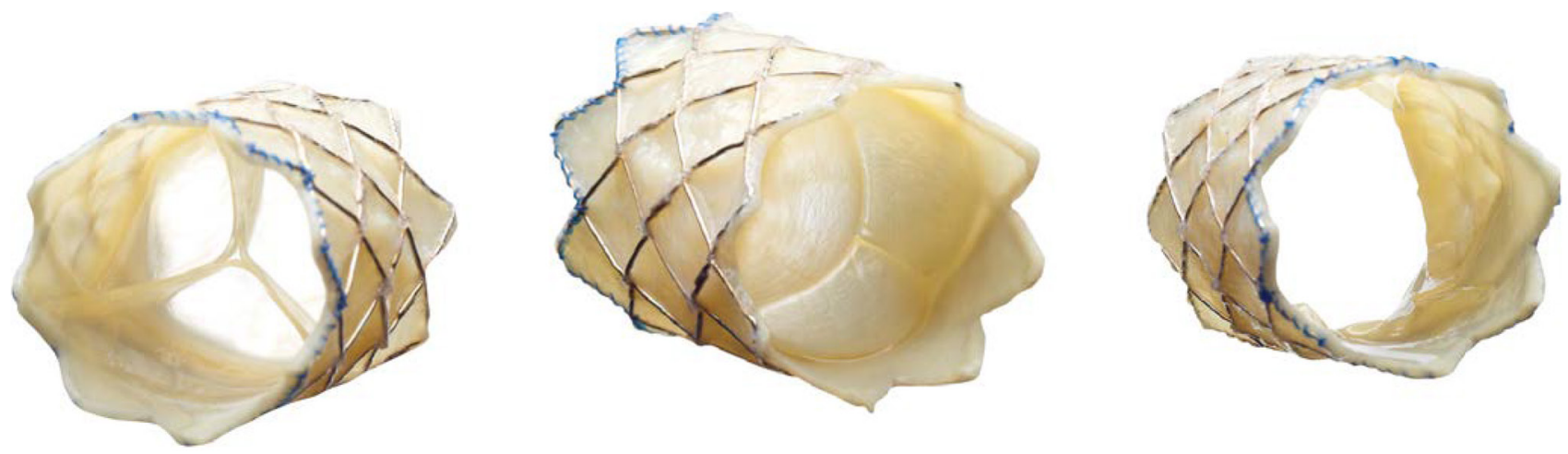

Figure 1 Depiction of the Medtronic Melody ${ }^{\circledR}$ transcatheter pulmonary valve $(18 \mathrm{~mm}$ bovine jugular vein valve with platinum iridium frame). It can expand to $24 \mathrm{~mm}$ external diameter with a $22 \mathrm{~mm}$ Ensemble ${ }^{\circledR}$ Delivery System. The length of the Melody ${ }^{\circledR}$ Transcatheter Pulmonary Valve varies between 26.2, 24.2 or $23 \mathrm{~mm}$ respectively, depending upon the size of the Ensemble ${ }^{\circledR}$ Delivery System used (18, 20, $22 \mathrm{~mm}$, respectively, Medtronic Inc., Minneapolis, MN, USA). For PTVI mostly the largest balloon delivery ensamble (22 mm) is used. PTVI, percutaneous tricuspid valve implantation.

(100 mg/day or $3-5 \mathrm{mg} / \mathrm{kg}$ ) is administered for 6 months. After 6 months, a TEE, a cardiac magnetic resonance imaging (cMRI), an exercise test, and a clinical examination are performed.

\section{Available percutaneous valves}

The Medtronic Melody ${ }^{\circledR}$ valve (Figure 1) system involves a balloon-expandable prosthesis. For PTVI only the larger $18 \mathrm{~mm}$ valve is chosen. If dilated to an internal diameter of $22 \mathrm{~mm}$, the outer diameter is $24 \mathrm{~mm}$. The bovine jugular vein is sutured into a bare metal $34 \mathrm{~mm} 8 \mathrm{zig}$ Cheatham platinum stent (Cheatham Platinum stent, NuMED Inc., Hopkinton, NY). Expanded to $22 \mathrm{~mm}$ internal diameter the stent shortens to a length of $24 \mathrm{~mm}$. The valve is hand crimped onto a $22 \mathrm{~mm}$ balloon-in-balloon delivery catheter (Ensemble Transcatheter Delivery System, Minneapolis, MN, USA) with a maximal outer diameter of $22 \mathrm{~F}$. If a shorter valve is needed, the distal and proximal zigs of the stent may be folded, adding additional $2 \mathrm{~mm}$ to the external diameter. The delivery system is then closed over the valve and the ensemble is guided over the wire into the final landing zone, where it can be re-opened, after which the $\mathrm{BiB}$ balloons are inflated.

The Edwards Sapien ${ }^{\circledR}$ valve (Figure 2) is a tri-leaflet bovine pericardial valve, hand sutured onto a rigid chromium-cobalt stent frame and is available in four sizes, 20, 23, 26 and $29 \mathrm{~mm}$ external diameter. Once dilated, the stent length ranges from $14.3 \mathrm{~mm}$ for the $23 \mathrm{~mm}$ valve to $19.1 \mathrm{~mm}$ for the $29 \mathrm{~mm}$ valve. The valve is crimped using a specially designed tool to mount it onto a $30-\mathrm{mm}$ long, noncompliant high-pressure balloon. The valve is delivered through a 14 or $16 \mathrm{~F}$ sheath into the femoral vein. The French size refers to the internal diameter of the expandable sheaths. The outer diameter of the Melody ${ }^{\circledR}$ valve is $22 \mathrm{~F}$, but it can be delivered through the Edwards $16 \mathrm{~F}$ e-sheath. Hence, the external diameters of the valves (Melody ${ }^{\circledR}$ and Sapien ${ }^{\circledR} 23,26,29 \mathrm{~mm}$ ) do not differ significantly. Once in the inferior vena cava, the valve is pushed forward onto the balloon. It is then advanced unguarded into the TV over the guide wire, where the valve is balloon dilated. Usually only the 26 and $29 \mathrm{~mm}$ Sapien valves are used for PTVI. With slight overfilling of the delivery balloon the Sapien $29 \mathrm{~mm}$ valve may be expanded to $31 \mathrm{~mm}$ (24).

\section{Comment}

PTVI is technically feasible, safe, and effective in selected patients with dysfunction of surgically implanted bioprostheses in TV position, using both Melody and Sapien transcatheter valves. Patients with congenital heart disease (CHD) and a failing bioprosthesis in tricuspid position usually have experienced several open-heart surgeries, present in a reduced clinical state and constitute a high-risk patient collective for repeated open-heart surgery. PTVI is a straight forward procedure with a high success rate and a low periprocedural complication rate. A recently published paper on surgical TV valve exchange in patients with CHD $(n=51)$ and bioprosthesis dysfunction documented a 30-day mortality rate of $9 \%$, with an 

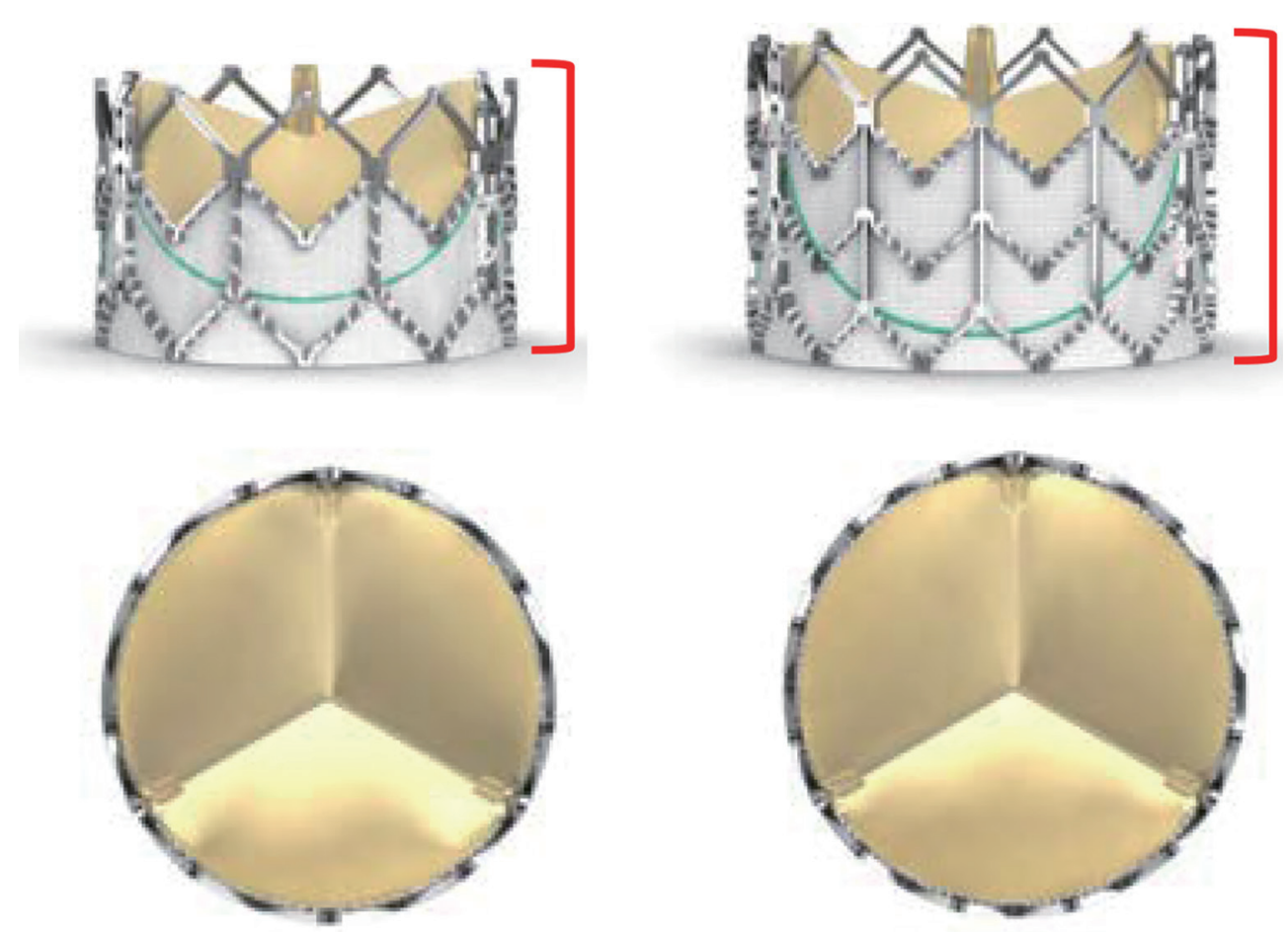

Figure 2 The Edwards Sapien ${ }^{\circledR}$ XT valve: trileaflet bovine pericardium valve hand-sutured in a stainless-steel stent. The length of the expanded Sapien $26 \mathrm{~mm}$ valve is $17.2 \mathrm{~mm}$ and of the Sapien $29 \mathrm{~mm}$ valve is $19.1 \mathrm{~mm}$. The valve is available in 20, 23, 26 and $29 \mathrm{~mm}$ external diameters. For PTVI mostly the 26 and $29 \mathrm{~mm}$ valves are used. PTVI, percutaneous tricuspid valve implantation.

estimated survival rate at 10 years of $80 \%$. Freedom from re-intervention at 1, 5 and 10 years was $100 \%, 86 \%$ and $81 \%$, respectively. However, after 10 years only $58 \%$ of patients did not show echocardiographic evidence of TV dysfunction, mostly TR was diagnosed (25).

Today, long term data on sustained preserved valve function after PTVI is not available, but short to medium term data are promising (17). In this study only one valve (Melody valve) had to be exchanged, 1.5 years after PTVI for recurrent severe TV regurgitation and all treated patients were alive. Histologically, the explanted Melody valve did not show any signs of valve infection or thrombosis, but the valve leaflets were thickened, with a central coaptation defect causing the valve regurgitation. The findings of this patient were published in a recent report by Bentham et al. (26). Notably, this patient subsequently developed early TV regurgitation (after six months) of the replaced surgical bioprosthesis (Carpentier-Edwards Perimount valve), and moderate regurgitation of a Sapien XT $29 \mathrm{~mm}$ valve implanted percutaneously within the bioprosthesis, suggesting that there was something about this patient's physiology/anatomy that predisposed to TV prosthetic valve dysfunction. The reason for this recurrent dysfunction of various biological valves (i.e., Melody, Perimount and Sapien XT 29) implanted surgically and with different transcatheter methods remains speculative. Severe RV dysfunction was present, which leads to slow almost continuous blood flow through the TV, such that the valve did not open and close in the normal phasic fashion, and proper valve leaflet function was not achievable. This is similar to the physiologic conditions after a Fontan procedure in which the initially suggested implantation of homograft valves proved to be unsuccessful (27).

The largest study on PTVI is from an international, multicenter registry study on 156 patients with bioprosthetic TV dysfunction of whom $71 \%$ were in New York Heart Association functional class III-IV. In 152 attempted procedures (Melody 94, Sapien 58) the valve was successfully implanted in 150 patients (99\%). The RA-RV inflow gradient was reduced significantly and TR was abolished. During follow-up (median 13.3 months), 22 patients had died, 5 within 30-days post PTVI; all had been in NYHA class III or IV and 9 had been hospitalized before PTVI. There were $10 \mathrm{TV}$ reinterventions, and 
three patients (including the patient mentioned above) had significant recurrent TV dysfunction. At follow-up, 77\% of patients were in NYHA class I or II $(\mathrm{P}<0.001$ versus before PTVI). The authors concluded that PTVI should be considered a viable option for treatment of failing TV bioprostheses (18).

Finally, first reports are published on PTVI in patients without a bioprosthesis in tricuspid position $(28,29)$.

In conclusion, patients with severe TV bioprosthesis dysfunction are a high-risk population for redo open heart surgery. In contrast to redo surgery, PTVI is far less invasive and short-to medium term follow-up results are promising and show similar hemodynamic results. PTVI performed by an experienced operator is a feasible, safe and effective treatment option in these patients, possibly reducing the total number of open-heart surgeries throughout their total lifetime. Further studies with larger patient numbers and longer follow-up are needed.

\section{Acknowledgements}

None.

\section{Footnote}

Conflicts of Interest: A Eicken is proctor for the Medtronic Melody valve; P Ewert is proctor for the Medtronic Melody valve and the Edwards Sapien valve.

\section{References}

1. Attenhofer Jost CH, Connolly HM, Dearani JA, et al. Ebstein's anomaly. Circulation 2007;115:277-85.

2. Rogers JH, Bolling SF. The tricuspid valve: current perspective and evolving management of tricuspid regurgitation. Circulation 2009;119:2718-25.

3. Topilsky Y, Nkomo VT, Vatury O, et al. Clinical outcome of isolated tricuspid regurgitation. JACC Cardiovasc Imaging 2014;7:1185-94.

4. Nath J, Foster E, Heidenreich PA. Impact of tricuspid regurgitation on long-term survival. J Am Coll Cardiol 2004;43:405-9.

5. Said SM, Dearani JA, Burkhart HM, et al. Management of tricuspid regurgitation in congenital heart disease: is survival better with valve repair? J Thorac Cardiovasc Surg 2014;147:412-7.

6. da Silva JP, Baumgratz JF, da Fonseca L, et al. The cone reconstruction of the tricuspid valve in Ebstein's anomaly.
The operation: early and midterm results. J Thorac Cardiovasc Surg 2007;133:215-23.

7. Lange R, Burri M, Eschenbach LK, et al. Da Silva's cone repair for Ebstein's anomaly: effect on right ventricular size and function. Eur J Cardiothorac Surg 2015;48:31620; discussion 320-1.

8. Hwang HY, Kim KH, Kim KB, et al. Propensity score matching analysis of mechanical versus bioprosthetic tricuspid valve replacements. Ann Thorac Surg 2014;97:1294-9.

9. Guenther T, Noebauer C, Mazzitelli D, et al. Tricuspid valve surgery: a thirty-year assessment of early and late outcome. Eur J Cardiothorac Surg 2008;34:402-9; discussion 409.

10. Baumgartner H, Bonhoeffer P, De Groot NM, et al. ESC guidelines for the management of grown-up congenital heart disease (new version 2010). Eur Heart J 2010;31:2915-57.

11. van Slooten YJ, Freling HG, van Melle JP, et al. Longterm tricuspid valve prosthesis-related complications in patients with congenital heart disease. Eur J Cardiothorac Surg 2014;45:83-9.

12. Bonhoeffer P, Boudjemline Y, Saliba Z, et al. Percutaneous replacement of pulmonary valve in a right-ventricle to pulmonary-artery prosthetic conduit with valve dysfunction. Lancet 2000;356:1403-5.

13. Eicken A, Fratz S, Hager A, et al. Transcutaneous Melody valve implantation in "tricuspid position" after a Fontan Bjork (RA-RV homograft) operation results in biventricular circulation. Int J Cardiol 2010;142:e45-7.

14. Tanous D, Nadeem SN, Mason X, et al. Creation of a functional tricuspid valve: novel use of percutaneously implanted valve in right atrial to right ventricular conduit in a patient with tricuspid atresia. Int J Cardiol 2010;144:e8-10.

15. Roberts PA, Boudjemline Y, Cheatham JP, et al. Percutaneous tricuspid valve replacement in congenital and acquired heart disease. J Am Coll Cardiol 2011;58:117-22.

16. Godart F, Baruteau AE, Petit J, et al. Transcatheter tricuspid valve implantation: A multicentre French studyImplantion percutanée d'une valve tricuspide: une étude muticentrique française. Archives of Cardiovascular Diseases 2014;107:583-91.

17. Eicken A, Schubert S, Hager A, et al. Percutaneous Tricuspid Valve Implantation - Two-Center Experience With Midterm Results. Circ Cardiovasc Interv 2015;8.

18. McElhinney DB, Cabalka AK, Aboulhosn JA, et al. Transcatheter Tricuspid Valve-in-Valve Implantation for 
the Treatment of Dysfunctional Surgical Bioprosthetic Valves: An International, Multicenter Registry Study. Circulation 2016;133:1582-93.

19. Shah AH, Horlick EM, Eicken A, et al. Transcatheter valve implantation for right atrium-to-right ventricle conduit obstruction or regurgitation after modified Björk-fontan procedure. Catheter Cardiovasc Interv 2017;89:298-305.

20. Taggart NW, Cabalka AK, Eicken A, et al. Outcomes of Transcatheter Tricuspid Valve-in-Valve Implantation in Patients With Ebstein Anomaly. Am J Cardiol 2018;121:262-8.

21. Cullen MW, Cabalka AK, Alli OO, et al. Transvenous, Antegrade Melody Valve-in-Valve Implantation for Bioprosthetic Mitral and Tricuspid Valve Dysfunction: A Case Series in Children and Adults. JACC Cardiovasc Interv 2013;6:598-605.

22. Gurvitch R, Cheung A, Ye J, et al. Transcatheter valve-invalve implantation for failed surgical bioprosthetic valves. J Am Coll Cardiol 2011;58:2196-209.

23. Piazza N, Bleiziffer S, Brockmann G, et al. Transcatheter aortic valve implantation for failing surgical aortic bioprosthetic valve: from concept to clinical application

Cite this article as: Eicken A, Ewert P. Percutaneous tricuspid valve implantation in failing bioprosthesis. Cardiovasc Diagn Ther 2018;8(6):765-770. doi: 10.21037/cdt.2018.09.08 and evaluation (part 1). JACC Cardiovasc Interv 2011;4:721-32.

24. Shivaraju A, Kodali S, Thilo C, et al. Overexpansion of the SAPIEN 3 Transcatheter Heart Valve: A Feasibility Study. JACC Cardiovasc Interv 2015;8:2041-3.

25. Burri M, Vogt MO, Hörer J, et al. Durability of bioprostheses for the tricuspid valve in patients with congenital heart disease. Eur J Cardiothorac Surg 2016;50:988-93.

26. Bentham J, Qureshi S, Eicken A, et al. Early percutaneous valve failure within bioprosthetic tricuspid tissue valve replacements. Catheter Cardiovasc Interv 2013;82:428-35.

27. Fontan F, Baudet E. Surgical repair of tricuspid atresia. Thorax 1971;26:240-8.

28. Navia JL, Kapadia S, Elgharably H, et al. First-in-Human Implantations of the NaviGate Bioprosthesis in a Severely Dilated Tricuspid Annulus and in a Failed Tricuspid Annuloplasty Ring. Circ Cardiovasc Interv 2017;10.

29. Navia JL, Baeza C, Maluenda G, et al. Transcatheter mitral valve replacement with the NaviGate stent in a preclinical model. EuroIntervention 2017;13:e1401-9. 